\title{
B2B App Store Governance in Software Platform Ecosystems: Dimensions and Types
}

\begin{tabular}{|c|c|c|}
\hline Rob Jago Floetgen & Ulrich Ziegler & Jörg Weking \\
\hline Technical & Celonis SE & Technical \\
\hline $\begin{array}{l}\text { University of } \\
\text { Munich }\end{array}$ & & $\begin{array}{l}\text { University of } \\
\text { Munich }\end{array}$ \\
\hline rob.floetgen & $\underline{\text { u.ziegler }}$ & joerg.weking \\
\hline @.tum.de & @ celonis.com & @,tum.de \\
\hline
\end{tabular}

\begin{abstract}
The ever-increasing customer demand for use casespecific $B 2 B$ software puts platform owners into a challenging situation where integrating a B2B app store into their digital platform becomes a necessity to manage the dynamics of software platform ecosystems. However, platform owners face uncertainty and experiment, while platform ecosystem research provides limited guidance for specific B2B app store governance. Closing this gap, we use multiple case studies and develop three taxonomies for architecture, control mechanisms, and demand generation to provide an overview of the solution space for $B 2 B$ app store governance. We further derive three robust $B 2 B$ app store governance types: platform play, transaction channel, and community platform. This paper enriches the B2C-driven and core-offering related research on digital platform governance with tangible B2B app store governance dimensions and types. We envision to guide practitioners in identifying and selecting governance characteristics to remain competitive and provide innovation for their B2B app stores.
\end{abstract}

\section{Introduction}

In the past decade, an ever-growing number of large business-to-business (B2B) software platforms such as Amazon Web Services or Microsoft Azure continued to proliferate proprietary online application marketplaces (e.g., B2B app stores) as "digital storefronts" on top of their digital platforms offering core products and services. Whereas Apple may have been responsible for acquainting "app store" as a portion of the general public and common vernacular, the godfather of the B2B app store is Salesforce's AppExchange which was launched back in 2005 [1] The concept of a B2B app store is to provide additional value to the platform owner's customers and enable third-party developers (e.g., independent software vendors) to distribute own-build software extensions;

\begin{tabular}{|c|c|c|}
\hline $\begin{array}{l}\text { Matthias Hoefler } \\
\text { Celonis SE }\end{array}$ & $\begin{array}{l}\text { Tobias Riasanow } \\
\text { Technical } \\
\text { University of } \\
\text { Munich } \\
\text { tobias.riasanow }\end{array}$ & $\begin{array}{l}\text { Markus Böhm } \\
\text { Technical } \\
\text { University of } \\
\text { Munich } \\
\text { markus.boehm }\end{array}$ \\
\hline celonis & a,tum.de & a,tum. \\
\hline
\end{tabular}

thereby complementing the in-house built application portfolio [2].

As platform owners increasingly realize that supplementary applications are critical to successfully manage the dynamics of software platform ecosystems [3], the reasons to launch a B2B app store become obvious: For example, B2B software platforms are often developed as a standard software solution that provides natural extension opportunities for integrating specific customer use cases. B2B app stores allow software platforms to provide their users with the expertise from their own and particularly their partners' best practices from domain specific implementations [4], which could shorten the typically long and resource-intensive B2B software sales cycles [5]. Moreover, software platforms utilizing B2B app stores might generate higher competitive differentiation through more solutions and higher adoption of their software platform, reducing churn [6].

However, continual governance adjustments of putative mature and also recently launched $\mathrm{B} 2 \mathrm{~B}$ app stores indicate that platform owners face uncertainty and are forced to experiment [7]. For example, the Execution Management platform vendor Celonis, who recently launched its EMS Store, is continually determining and optimizing the governance characteristics of its B2B app store based on recent user adoption and interactions with complementors [4].

Existing research on platform ecosystem governance, e.g., network effect governance [8], traditional pricing [9], platform openness [10], or boundary resources [11] provides a fundamental understanding that can be conveyed toward specific B2B app store governance. While these studies have been vital in advancing a holistic understanding about the governance of platform ecosystems [12], these frameworks are either too narrowed (e.g., related to the core offering of the digital platform [8]), too specific (e.g., focusing on $\mathrm{B} 2 \mathrm{C}$ markets [13] or only focus on few governance dimensions [10]), incomplete (e.g., not considering recent originated characteristics [14]), or too general (e.g., not deriving overreaching and robust 
governance types [15]) to classify the rapidly developing and changing nuances required to explain the complex mechanisms of B2B app store governance. In sum, the existing literature yields only sparse and tangible conceptual guidance concerning our research question: What are the B2B app store governance dimensions and types in software platform ecosystems?

To evaluate, organize, and understand this complex domain, taxonomies might be appropriate [e.g., 14]. Taxonomies constitute a "form of classification," i.e., a "conceptually or empirically derived grouping" that enables researchers and practitioners to structure a complex domain [16]. Taxonomies further assist in deriving robust overreaching types, as they may reveal unique building blocks of B2B app store governance $[15,17]$.

Building on taxonomy development, we connect the knowledge from existing research and empirical data, i.e., ten interviews and 2180 pages of secondary data, such as partner documents. We derive recurring governance dimensions and tangible characteristics through constant comparison and visualize them in three taxonomies, i.e., architecture, control mechanisms, and demand generation. Aggregating the repeated analyzed combinations of characteristics, we identify three robust governance types for B2B app stores. Thus, we combine and structure the fragmented knowledge across platform ecosystem governance toward specific B2B app store governance and provide decision support when designing a B2B app store. Finally, we discuss our findings considering the lessons learned from our interview partners through designing app stores, as well as our theoretical contribution to the research field.

\section{Theoretical Background}

A substantial body of IS research has examined digital platforms and their ecosystem from multiple perspectives $[12,18]$. From a technical perspective, digital platforms are defined as an extensible codebase on which third-party developers can develop complementary products and services through the use of interfaces [19]. In our research, we follow the sociotechnical definition of platform ecosystems to empirically study the mechanisms for orchestrating a B2B app store where "a platform owner [...] implements governance mechanisms to facilitate valuecreating mechanisms on a digital platform between the platform owner and an ecosystem of autonomous complementors and consumers" [20]. Following the three dimensions "providing autonomy", "ensuring integration", and "creating incentives" of platform ecosystem governance by Tiwana [19], previous research provides a decent understanding of relevant governance mechanisms to orchestrate a digital platform ecosystem [15]. For example, a concept relevant to platform ecosystem governance is providing boundary resources through APIs, SDKs, and other development interfaces that enable and facilitate complementors to co-develop solutions on the platform [21]. The concept of openness describes a relevant platform ecosystem governance mechanism to limit the use, development, and commercialization of solutions shared on the platform [10]. The concept of pricing and revenue sharing in platform ecosystem governance addresses monetization streams in the ecosystems and how they influence network effects [9].

By integrating app stores into the concept of digital platforms [13] we use the term "product platform" to refer to the core offering of the digital platform (e.g., Microsoft Azure) and the term "app store" (e.g., Azure Marketplace) to designate the digital interface between the platform owner and the stakeholders in the platform ecosystem (Figure 1) [2]. Platform owners implement app stores to create a venue for the simplified exchange

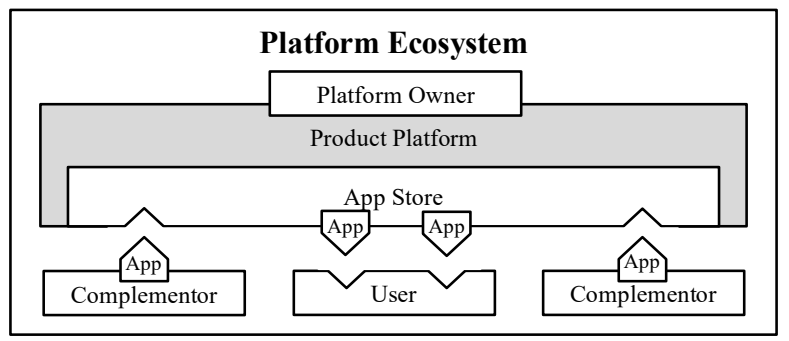

Figure 1: Platform Ecosystem Architecture with App Store

of solutions between third-party developers and end users [22]. Given the inherently high fragmentation of complementors and users, app store ecosystems provide a healthy environment for ecosystem participants and lead to a high number of platform-specific applications [23]. App stores offer both complementors and end users a novel environment to develop and procure software that differs from previously used channels [22]. However, the experience can influence the perceived trust in the platform owner-complementor relationship [24]. To date, few studies on platform governance have integrated concepts of novel expectations and trust through the implementation of app stores. As a basic functionality, payment and commissions are enabled through app stores [25]. Previous implementations of app stores have shown innovative forms of monetization for both complementors and users, e.g., development fees, upfront commitment to a certain level of usage, or paid ancillary services [26]. Still, the current literature on platform governance focuses on traditional pricing strategies, such as revenue sharing and subsidizing complementors; thus, specific, relevant metrics are not considered [27]. The implementation of app stores 
offers complementors and users new opportunities for co-creating value [28]. Consequently, to encourage complementors to align with the platform's strategic and operational objectives, input control becomes an essential concept in platform governance [29]. Furthermore, newly created partner programs for different types of complementors facilitate participation in the app store ecosystem; however, such programs require an effective mechanism for the validation and allocation of partners [30]. Yet, research on platform ecosystems in terms of novel characteristics, such as B2B app store experience, pricing, and partner programs remains sparse.

Building on the concepts associated with platform ecosystems, an extensive body of IS research on mobile app stores has emerged that attempts to understand the underlying concepts that drive the success of B2C app stores [31]. Existing implementations, such as the Apple App Store and Google Play Store, have become commonly used empirical cases to investigate value cocreation within a large ecosystem of users and complementors [32]. In such B2C-based app stores, research found that the degree of control over developer autonomy correlates with the quality and productiveness of the overall ecosystem [33]. Another study on B2C app stores highlights the importance of the quality assurance mechanisms exercised by the platform owner for submitted applications, as users are not willing to search and pay for apps of unknown quality [2].

However, B2B differ from B2C markets. As B2B app store users often have IT and business-related backgrounds, the buying process is different because whole departments rather than a single person are responsible for buying products or services in companies. Thus, pricing methods need to be flexible and cannot simply be based on approaches used with B2C app stores. Another distinguishing feature of B2B app stores compared to $\mathrm{B} 2 \mathrm{C}$ stores is the increased expectation of users concerning the quality of the apps, as prices are significantly higher and the applications are often used in production software systems, where reliable operation is critical to the entire system. Furthermore, a B2B app store attracts commercially motivated complementors, e.g., independent software vendors, original equipment manufacturers, or consultants with whom further go-to-market motions are planned on top of the solution and access to the sales base is given. In contrast, B2C app stores have both private and commercial users and offer predominantly standardized demand generation packages, which are not sustainable in the B2B domain. It is thus unclear, under which conditions a digital platform with an app store can be successful in B2B ecosystems.

As the number and importance of B2B app stores increases, a detailed overview of the specific governance concepts for B2B app stores is required. Although the current literature on platform governance provides a profound understanding of mechanisms to orchestrate a platform ecosystem, relevant concepts that specifically address the novel features and tangible characteristics of B2B app stores have not been considered. Existing research provides frameworks to classify high-level governance concepts [17]; however, granular analyses that could guide practical app store implementations are lacking. Even the more specific studies on B2C app stores do not consider numerous characteristics that are relevant to $\mathrm{B} 2 \mathrm{~B}$ app stores and therefore cannot be used to infer governance principles from the $\mathrm{B} 2 \mathrm{C}$ to the $\mathrm{B} 2 \mathrm{~B}$ domain. A general overview of governance concepts for B2B app stores that combines relevant theory on platform governance with practical insights from successful app store implementations is required.

\section{Methodology}

This work follows a three-phase research approach. First, we created the empirical basis of this study with multiple case study research based on Yin [38] and coded the cases based on Corbin et al. [34]. This rich case study data provides the basis for the second phase: the development of a detailed taxonomy and types. We apply the method proposed by Nickerson et al. [16] to systematically build and evaluate a taxonomy for B2B app store governance. This method facilitates combining theoretical concepts about platform ecosystem governance with empirical findings from the multiple B2B app store case study. The third phase follows the approach of Punj et al. [35] to cluster and derive robust $\mathrm{B} 2 \mathrm{~B}$ app store governance types.

\subsection{Multiple Case Study}

First, following the method proposed by Yin [36], we performed a multiple case analysis with ten cases, as shown in Table 1. Each case in the database represents a B2B app store implementation in the enterprise software domain. The criteria used to choose the cases were selected to provide sufficient information on app store governance from cases that have a large number of active users and complementors [37]. Second, following the guidelines provided by Gläser et al. [38], we conducted ten semi-structured interviews with the case vendors and triangulated the data with 2180 pages of partner-related documents that were retrievable through the vendors' websites [36]. The selected interviewees are either working in a leading strategic position or had ownership stakes in the app store, who have privileged 
Table 1: Interviews and documents from the case study

\begin{tabular}{|c|c|c|c|c|c|c|c|}
\hline$\#$ & Case & Vendor & Market & Interviewee Role & Duration & Sec. Documents Pages & Exemplary Reference \\
\hline In1 & Appian AppMarket & Appian & BPM & App Store Owner & $51: 40 \mathrm{~h}$ & 60 & [39] \\
\hline In 2 & AWS Marketplace & AWS & Cloud & ISV Manager & $58: 47 \mathrm{~h}$ & 285 & [26] \\
\hline In 3 & Azure Marketplace & Microsoft & Cloud & Cloud Architect & $55: 46 \mathrm{~h}$ & 831 & [40] \\
\hline In4 & Blue Prism Digital Exchange & Blue Prism & RPA & App Store Owner & $99: 38 \mathrm{~h}$ & 8 & [41] \\
\hline In5 & Celonis EMS Store & Celonis & EMS & ISV Manager & $47: 32 \mathrm{~h}$ & 34 & {$[42]$} \\
\hline In6 & Pega Marketplace & Pega & BPM & ISV Manager & $76: 08 \mathrm{~h}$ & 6 & [43] \\
\hline $\operatorname{In} 7$ & Salesforce AppExchange & Salesforce & CRM & ISV Manager & $92: 34 \mathrm{~h}$ & 90 & {$[30]$} \\
\hline In8 & SAP Store & SAP & ERP & Digital Sales & $60: 47 \mathrm{~h}$ & 27 & [44] \\
\hline In9 & ServiceNow Store & ServiceNow & ERP/CRM & Solution Engineer & $53: 02 \mathrm{~h}$ & 22 & {$[45]$} \\
\hline In10 & Splunkbase & Splunk & Analytics & Product Manager & $76: 37 \mathrm{~h}$ & 817 & [46] \\
\hline
\end{tabular}

access to information and knowledge on the respective B2B app store (Table 1). Third, following the method proposed by Corbin et al. [34], we applied an iterative coding approach to the interview results with open, axial, and selective coding to identify governance characteristics in the interview quotes and identify relationships between the characteristics.

\subsection{Taxonomy Development}

Following the approach adopted by Nickerson et al. [16], we performed three iterations with alternating inductive and deductive cycles to develop and evaluate the taxonomy on B2B app store governance. In addition, we specified objective and subjective ending conditions to terminate the iterative process [16]. Before starting, we adopted the governance dimensions of Tiwana [19] with insights from our first research phase (3.1) to structure B2B app store governance toward the three sub-taxonomies of architecture, control mechanisms, and demand generation to better guide the selection of governance dimensions. For each sub-taxonomy, the following three iterations were performed:

The first iteration follows the conceptual-toempirical approach and builds an initial taxonomy for B2B app store governance connecting general B2B app store literature. Considering the rigor of the defined characteristics, we analyzed the literature for existing governance dimensions and characteristics and synthesized relevant concepts into an initial taxonomy. For example, the governance concept of "External Relationship Management" was considered to attribute for the management of complementors in the digital platform ecosystem [14]. Due to the limited number of publications on B2B app store literature, we expanded our initial search to include research on platform ecosystem governance, where app stores are not part of the platform. The more generalist nature of the governance concepts for platform ecosystems allows some concepts to be re-applied and referenced to platform ecosystems with app stores. The second iteration follows an empirical-to-conceptual approach and further develops the initial taxonomy with empirical aspects from the multiple case studies. For example, the initial concept of "External Relationship Management" evolved into the dimensions "Supervision Roles" and "Supervision Engagements" of the control mechanisms taxonomy. We classified nine app stores from our sample with the taxonomy, added further characteristics that were derived from the coded results, grouped the characteristics into dimensions, and revised the taxonomy [16]. Ultimately, we performed this process until all cases were included. In the third iteration, we applied an empirical-to-conceptual approach to evaluate the resulting taxonomy by exposing it to a tenth case that was not used in the previous inductive step. The case was fully representable by the dimension and characteristics defined in the taxonomy.

\subsection{Qualitative Cluster Analysis}

In the third phase, we applied the resulting B2B app store governance taxonomy to the ten cases by following a within-case analysis approach [36]. As part of this, we conducted a qualitative cluster analysis in a cross-case setting and applied constant comparisons to the resulting types. Ultimately, we were able to identify three robust B2B app store governance types that share common governance expressions within their case group. The derivation of robust types is based on the relative occurrence of governance characteristics within the group of a type. For example, the Platform Play governance type is characterized by the consistent occurrence of the characteristic "High Verification" for the "QA Rigor Level" dimension. Each type has different centers along the dimensions and characteristics in the taxonomy [36]. To ensure clarity and singularity among the types, we pruned the taxonomy on dimensions where there was no singular identifiable expression. 


\section{Results}

\subsection{B2B App Store Governance Taxonomy}

The resulting taxonomy on B2B app store governance is divided into three sub-taxonomies that relate to architecture, control mechanisms and demand generation (Tables 2, 3 and 4). Dimensions marked with an asterisk (*) are mutually exclusive and can only be defined through a single characteristic, and dimensions without an asterisk are not mutually exclusive and can be defined by a combination of multiple characteristics.

The first sub-taxonomy on architecture covers the infrastructure and solution-related governance components and resources that form the fundamental and operational basis of each app store (Table 2). The concept Solution describes the types of solutions that are distributed through the app store and how they relate to the product platform. The concept Infrastructure relates to the governance of app store infrastructure, i.e., the different portals and components that characterize the usability of the app store. The Technical concept addresses all dimensions related to the technical functionality of an app store and the solution types offered. The Resources concept describes the tools provided to complementors to facilitate the development of solutions and enable complementors to use the platform elements.

The second sub-taxonomy on app store governance comprises aspects related to control mechanisms, which establish operating principles and exert fundamental directives on all participants in the ecosystem (Table 3). The concept of Openness defines which groups have access to the app store and can participate in the ecosystem to create or receive value. The concept of Complementor Selection defines which type of complementors participate in the app store ecosystem and how they are selected. By defining the concept of Complementor Management, the supervision through assigned roles is specified. The concept of Input Control is implemented in app store governance to validate and ensure a certain level of quality of the content offered through the store. The concept of Monitoring provides feedback about the complementors solutions. The concept of Complementor Monetization defines the mechanisms that app store owners implement to monetize complementors. The concept of End user Monetization describes the monetary mechanisms used to price the use of the app store and the available solutions.

The third sub-taxonomy, i.e., demand generation, describes the governance principle employed to incentivize complementors and end users to participate, contribute, and consume content through the app store (Table 4): The concept of Marketing defines the components used to incentivize partners and end users to engage on the app store. The concept of Sales describes the method used to sell content through adjunct channels. The concept of Feature describes further incentives for partners and how end users are targeted to consume through the app store.

Table 2: Architecture Taxonomy (1=AWS Marketplace, 2=Salesforce AppExchange, 3=Appian AppMarket)

\begin{tabular}{|c|c|c|c|c|c|c|c|c|}
\hline $\begin{array}{l}\text { Con- } \\
\text { cept }\end{array}$ & Dimension & \multicolumn{7}{|c|}{ Characteristics } \\
\hline \multirow{5}{*}{ 号 } & Solution Type & \multicolumn{2}{|l|}{$\mathrm{SaaS}^{2}$} & \multicolumn{2}{|c|}{ Application $1,2,3$} & \multicolumn{2}{|c|}{ Use Case $^{1,2,3}$} & Service $^{2}$ \\
\hline & Application Types & Platform App ${ }^{1,2}$ & & Modules $^{1,3}$ & \multicolumn{2}{|c|}{ Connectors $^{2,3}$} & Templates $^{2,3}$ & Dashboard $^{1,3}$ \\
\hline & Integration Type & \multicolumn{2}{|c|}{ Platform Native ${ }^{1,3}$} & \multicolumn{2}{|c|}{ Native + Integration ${ }^{1,3}$} & \multicolumn{2}{|c|}{ Integration $^{1,3}$} & Hosting $^{2}$ \\
\hline & Application Packaging* & \multicolumn{4}{|c|}{ Add-On ${ }^{1,2,3}$} & \multicolumn{3}{|c|}{ Standalone $^{3}$} \\
\hline & Application Customization* & \multicolumn{3}{|c|}{ Full $^{3}$} & \multicolumn{2}{|c|}{ Modular $^{1,2}$} & \multicolumn{2}{|c|}{ Not Allowed } \\
\hline \multirow{7}{*}{ 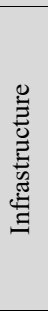 } & Portal Types & App Store Portal & $1^{1,2}$ & Partn & er Portal $1,2,3$ & Developer Po & rtal $1,2,3$ & User Portal $^{2}$ \\
\hline & Developer Portal Location* & \multicolumn{4}{|c|}{ On App Store ${ }^{3}$} & \multicolumn{3}{|c|}{ On Home Page ${ }^{1,2}$} \\
\hline & App Store Location & \multicolumn{3}{|c|}{ Dedicated Website $^{1,2}$} & \multicolumn{2}{|c|}{ Developer Portal $^{3}$} & \multicolumn{2}{|c|}{ Product Platform } \\
\hline & Application Filters & Type ${ }^{1,2,3}$ & \multicolumn{2}{|c|}{ Free/Paid $^{1,2,3}$} & Author $^{2,3}$ & Badges $^{2,3}$ & Industry 2,3 & Business $^{2}$ \\
\hline & Submission Account* & \multicolumn{3}{|c|}{ Complementor Account 1,2} & \multicolumn{2}{|c|}{ User Account } & \multicolumn{2}{|c|}{ No Account Required ${ }^{3}$} \\
\hline & Submission Location & \multicolumn{3}{|c|}{ App Store Portal ${ }^{2}$} & \multicolumn{2}{|c|}{ Partner Portal ${ }^{1,2}$} & \multicolumn{2}{|c|}{ Website $^{3}$} \\
\hline & Lead Destination & \multicolumn{2}{|c|}{ Partner Portal 1,2,3 } & \multicolumn{2}{|r|}{$\mathrm{CRM}^{1,2}$} & \multicolumn{2}{|c|}{ HTTPS Endpoint $^{2}$} & Mail \\
\hline & Software Coding Effort* & Code $^{1,}$ & 2,3 & & Low-C & $\operatorname{Code}^{2,3}$ & No-C & Code $^{1}$ \\
\hline 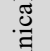 & Application Fulfillment & & App $S$ & tore $^{1,3}$ & & Com & plementor Landing & Page $^{2}$ \\
\hline 릉 & Application Deployment & Cloud & Envi & onment ${ }^{1}$ & $1,2,3$ & & On-Prem Environm & \\
\hline & Application Installation* & & Auton & atic $^{1,2}$ & & & Manually $^{3}$ & \\
\hline \& & Development Environment* & Offline ID & $\mathrm{DE}^{1,2}$ & & Online & $\mathrm{eIDE}^{1}$ & No & $n e^{3}$ \\
\hline$\Xi$ & Development Tools & API $1,2,3$ & Libr & ries $1,2,3$ & SDK $^{1,2,3}$ & Data Models $^{2}$ & Components $1,2,3$ & Semantic Layer $^{2}$ \\
\hline$\stackrel{\mathscr{Q}}{\simeq}$ & Developer Enablement & Documentation $^{1,2,3}$ & Tut & rials ${ }^{1,2}$ & Guidelines $^{1,2,3}$ & Sample Code ${ }^{1,2}$ & Use Cases $^{1,2}$ & Community $1,2,3$ \\
\hline
\end{tabular}


Table 3: Control Mechanisms Taxonomy (1=AWS Marketplace, 2=Salesforce AppExchange, 3=Appian AppMarket)

\begin{tabular}{|c|c|c|c|c|c|c|c|c|c|c|}
\hline $\begin{array}{l}\text { Con- } \\
\text { cept }\end{array}$ & Dimension & \multicolumn{9}{|c|}{ Characteristics } \\
\hline & App Store Accessibility* & \multicolumn{3}{|c|}{ Public ${ }^{1,2,3}$} & \multicolumn{4}{|c|}{ Customers $^{3}$} & \multicolumn{2}{|c|}{ Individual $^{3}$} \\
\hline$\stackrel{\mathscr{O}}{\Xi}$ & Developer Portal Users* & \multicolumn{3}{|c|}{ Developers $^{2}$} & \multicolumn{4}{|c|}{ Customers } & \multicolumn{2}{|c|}{ Shared ${ }^{1,3}$} \\
\hline ల్ & Application Availability & \multicolumn{3}{|c|}{ Public $1,2,3$} & \multicolumn{4}{|c|}{ Private $^{2}$} & \multicolumn{2}{|c|}{ Custom } \\
\hline & Geographic Targeting & \multicolumn{5}{|c|}{ All Countries $^{2,3}$} & \multicolumn{4}{|c|}{ Selected Countries ${ }^{1,2}$} \\
\hline \multirow{5}{*}{ 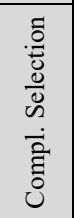 } & Complementor Types & \multicolumn{2}{|c|}{ Commercial Partners $1,2,3$} & \multicolumn{3}{|c|}{ Platform Owner $^{1,2,3}$} & \multicolumn{2}{|c|}{ Employees $^{3}$} & \multicolumn{2}{|c|}{ Customers $^{2,3}$} \\
\hline & Commercial Partner Types & Ind. Soft. Vend. 1,2,3 & Man. Ser & Prov. $^{2,3}$ & Res & 11 er ${ }^{1,2}$ & System In & grator $^{1,2}$ & $\mathrm{OEM}^{1,2}$ & Service Provider $^{2}$ \\
\hline & Complementor Entities & \multicolumn{3}{|c|}{ Company ${ }^{1,2,3}$} & \multicolumn{4}{|c|}{ Individual $^{3}$} & \multicolumn{2}{|c|}{ Anonymous } \\
\hline & Selection Criteria & Product $^{1,3}$ & \multicolumn{2}{|c|}{ Business Plan ${ }^{1}$} & Custo & er Base & \multicolumn{2}{|c|}{ Competitor } & Compliance $^{2}$ & Strategic Fit $^{1}$ \\
\hline & Commitment Criteria & \multicolumn{2}{|c|}{ Terms \& Conditions ${ }^{1,2,3}$} & \multicolumn{3}{|c|}{ Marketing Guidelines } & \multicolumn{2}{|c|}{ Seller Guidelines } & \multicolumn{2}{|c|}{ Frequent Update $^{1}$} \\
\hline \multirow{4}{*}{ 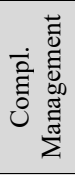 } & Partnership Tiers* & Multiple T & $\operatorname{Ciers}^{1,2}$ & & & $\operatorname{Sin}$ & gle Tier & & & her $^{3}$ \\
\hline & Supervision Roles* & Partner Mngr. ${ }^{1,2,3}$ & $\mathrm{O}_{1}$ & ations $\mathrm{N}$ & Mgr. $^{2}$ & Market & ing Mgr. ${ }^{1}$ & System I & Engineer $^{1}$ & Other \\
\hline & Supervision Engagement* & High Tou & $\mathrm{uch}^{1}$ & & & Medi & m Touch & & Low & $\operatorname{ouch}^{2,3}$ \\
\hline & Support Responsibility & Pla & tform $\mathrm{C}$ & $e^{2,3}$ & & & & Com & iplementor ${ }^{1,2,3}$ & \\
\hline & Targeted Product Maturity & Ready-to- & Sell $^{1,2}$ & & & & oncept $^{1}$ & & Early & Idea $^{3}$ \\
\hline $\bar{O}$ & App Selection Criteria & Market Size $^{1}$ & Use & $\operatorname{ase}^{1,3}$ & Platfo & $\mathrm{m} \mathrm{Fit}^{1,2}$ & $\mathrm{Co}$ & & Time-to-market & Contract Value \\
\hline है & Product Novelty* & Strict Nov & velty $^{2}$ & & & Reuse C & Component & & Not $\mathrm{C}$ & Checked \\
\hline 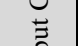 & QA Rigor Level* & High Verific & cation $^{1,}$ & & & Low V & erification & & No Ve & rification \\
\hline 气 & Verification Levels & Technical $^{1,2,3}$ & & ecurity ${ }^{1,}$ & & Func & ional $^{1}$ & & $\mathrm{JI}$ & Content \\
\hline & Verification Criteria & Compatibility $^{1,2,3}$ & Comp & eteness $^{1}$ & Perf & imance & IP Ow & ership & Policies & Expertise \\
\hline.$\Xi \stackrel{0}{\Xi}$ & App Store Statistics & Orders $^{1,2}$ & Usc & & Page & Visits $^{2}$ & Unique & isitors & Revenue $^{1,2,3}$ & KPIs $^{1,2}$ \\
\hline$\sum^{0} \overline{0}$ & Customer Feedback & Rating $1,2,3$ & & & eview ${ }^{1}$ & & & $\operatorname{tact}^{1,2}$ & Fea & ture Request \\
\hline$\dot{\vec{\pi}}$ & Monetization Model* & Revenue Sh & $\operatorname{are}^{1,2,}$ & & & App Pu & lishing $\mathrm{F}$ & & & Ione \\
\hline 言: & Listing Transaction* & & ransacta & $1,2,3$ & & & & Non- & Transactable ${ }^{1,2}$ & \\
\hline$\dot{\Sigma} \sum_{1}^{0}$ & Development Fees & Paid & & & & Memb & ership Fee & & & $1,2,3$ \\
\hline & Pricing Model & One-Time Fee & Subscri & $\mathrm{on}^{1,2,3}$ & & ric & Bring y. or & license $^{2}$ & Free-Trial $^{2}$ & Free $^{1,2,3}$ \\
\hline ๘ & Consumption Metric & \# of Users ${ }^{1,2,3}$ & & Hosts & & & $\operatorname{ata}^{2}$ & Band & width $^{2}$ & Time \\
\hline$\stackrel{\mathscr{N}}{\mathscr{N}}$ & Metric Selection* & Single Me & $\operatorname{tric}^{1,3}$ & & & Multip & e Metrics & & & Fee \\
\hline$\frac{1}{0}=$ & Consumpt. Commitment & & Upfro & & & & & Pay & as you go $1,2,3$ & \\
\hline$\sum$ & Discounting* & Volume Disc & counts $^{1}$ & & & Reseller & Discounts & & & one ${ }^{3}$ \\
\hline & Pricing Transparency* & Full Pric & $\mathrm{ce}^{1,2}$ & & & & Range & & Not & Visible $^{3}$ \\
\hline
\end{tabular}

Table 4: Demand Generation Taxonomy (1=AWS Marketplace, 2=Salesforce AppExchange, 3=Appian AppMarket)

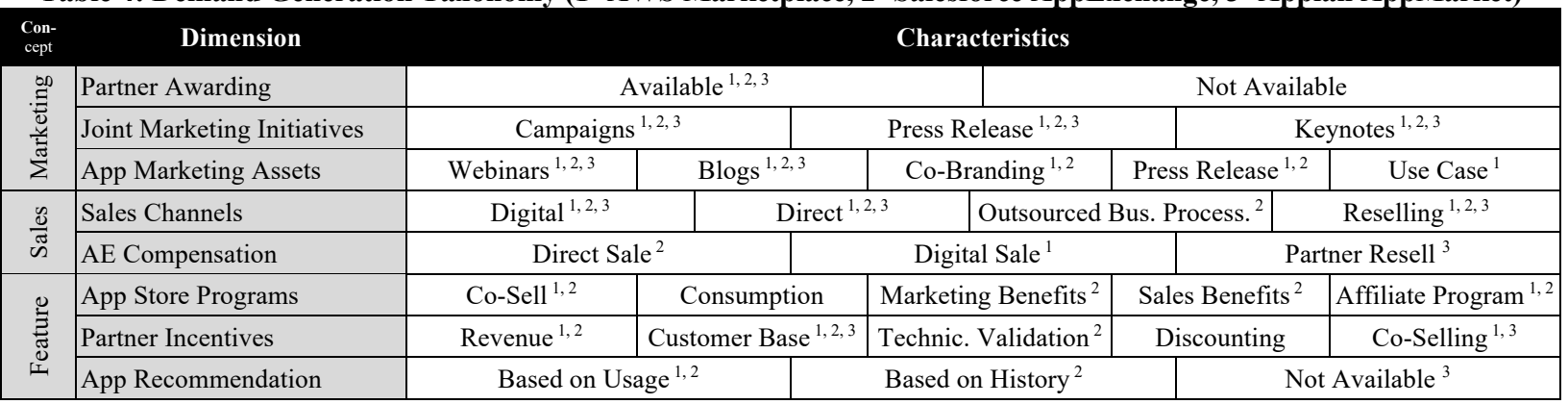

\subsection{B2B App Store Types}

Applying qualitative clustering reveals three robust app store governance types, i.e., Platform Play, Transaction Channel, and Community Platform.
4.2.1. Platform Play. The first governance type is represented by the Blue Prism Digital Exchange, Celonis EMS Store, Salesforce App Exchange, SAP Store, Service Now Store and Splunkbase in the examined sample. We exemplified the classification of the Salesforce AppExchange by a superscript "1" in 
the taxonomy. For these stores the underlying governance principle is to extend the product platform with a significant number of innovative and purposebuilt applications. App store owners run a Platform Play app store to "increase the adoption of the core offering $[\ldots]$, enhance the stickiness of the platform [...] [and] differentiate the offering of that from competitors" [In1-In10]. Platform Play app store owners attempt to capture and retain end users by offering a diverse set of value-enhancing solutions that differentiates their platform and ecosystem from competitors. For example, the SAP Store offers a variety of add-ons and extensions that enhance core functionality and increase user productivity. Thus, the user becomes dependent on these solutions and ultimately on the app store/platform because, in most cases, the products are only offered on one app store. The guiding principle of the Platform Play app store emphasizes ease of use for the end user by handling the fulfillment process entirely through the app store and automatically installing and deploying the procured solutions in the end user's cloud or on-prem environment. Complementors provide solutions that build natively on the platform and extend the core offering with third-party integrations. Platform owners provide developers with a variety of tools, e.g., SDKs, low-code application builders, to internalize external innovation potential. For example, the Salesforce AppExchange provides an API for solution integrations via Apex code and offers a native lowcode builder that enables partners to create workflows and list them on the AppExchange. According to an app store owner, the complementors are ranked in multiple partner tiers "[...] to provide incentives to increase their engagement [in the app store] by getting certified and participating in co-selling motions" [In1In10]. The onboarding and development process for a complementor is defined through a high touch onboarding experience by having the relationship owned by a partner manager, being supported in the development by a system engineer, and receiving marketing support from a marketing manager. The challenge for the app store owner is to balance innovation and control, as they set up a strict quality assurance policy that guarantees compliance with the core offering and increases usability for the end user. The input is controlled on technical, security, and functional levels, whereas the content is reviewed by end users. This creates an additional incentive for partners to deliver high-quality solutions as the open feedback culture circles back to their products. To attract a significant number of complementors, the monetization model for the complementor was simple in all app stores studied; product adoption was favored over direct revenue. A characteristic of the Platform
Play type is to increase consumption of the main product by offering solutions that extend the core offering through the app store and hence increase usage and indirect revenue. The complementor receives a free development instance and shares a fixed part of the revenue generated through the listing with the app store owner. Among all studied app stores, the end user is presented with a set of different pricing models, e.g., one-time fees, subscription, and metric-based pricing. In all cases, the app store also provided free software, and five out of six offered free trial versions of paid software.

4.2.2. Transaction Channel. The Azure Marketplace and the AWS Marketplace represent the Transaction Channel type through which a variety of solutions and services are distributed. We exemplified the classification of the AWS Marketplace by a superscript "2" in the taxonomy. This type is characterized by offering non-platform native solutions that are hosted on their infrastructure alongside solutions that also extend the core offerings of the platform. For example, both AWS Marketplace and Azure Marketplace host solutions that do not extend their core solutions. Complementors use this type of app store to increase their pipeline conversions and leverage co-sell motions offered in joint go-tomarket programs. In both cases, the underlying platform is a cloud vendor that hosts third-party solutions together with extensions to the app store's core offerings. Ensuring seamless integration, an app store owner confirmed that "most of the solutions offered on [the app store] are transactable, which allows them to be billed directly via [the app store]" [In1-In10]. Typically, the Transaction Channel type lists the greatest variety of solution types, such as SaaS offerings, applications, use cases, and professional services. It is the only type to offer services. The available applications are commonly packaged as standalone and can be procured as a subsidiary and extension of the product platform. The infrastructure offers dedicated portals for each app store management and to complete partner-related go-tomarket activities, as well as dedicated developer portals with a great number of resources. The development tools provided typically include APIs, libraries, SDKs, data models, components, and semantic layers, such as those offered in the AWS Marketplace. This type of app store accepts a variety of commercial partner types and is usually does not apply strict criteria in selecting partners. Product platform customers can act as ISVs and can represent managed service providers, resellers, solution integrators, and original equipment manufacturers. The onboarding process is generic and standardized with a great range of resources available for self- 
education. The input is rigorously controlled, and to onboard complementors offerings, products need to pass several quality assurance levels, including customer experience. Users use the Transactional Channel to find, test, and purchase third-party software for production purposes. In the two app stores studied in this category, the user had previously committed to a certain amount of consumption on the product platform and, according to an app store owner, "[...] procures software through [the app store] that counts against their consumption commitment" [In1In10]. The user is presented with a large variety of pricing metrics as the solutions offered can contain several features and components that are priced independently, and the hosting of the solution is billed separately from the core offering. Demand among users is created by leveraging different app store programs, press releases, and speeches on key features of new programs listed on the app store.

4.2.3. Community Platform. The third governance type, the Community Platform, is represented by the Appian AppMarket and the Pega Marketplace and creates a vivid ecosystem of customer-based complementors to contribute to the product platform. We exemplified the classification of the Appian AppMarket by a superscript " 3 " in the taxonomy. In all examined app store implementations, the external expertise is considered as internal expertise that is productized and listed on the app store by partners, customers or employees. The solutions offered are applications and use cases that comprise templates, modules, or connectors. It is expected that "customers act as complementors as they have gained [...] industry-specific expertise through applying [the core offering] in a day-to-day setting" [In1-In10] and contribute to the community of the platform by providing the insights. This is common practice with the Appian AppMarket, where actual customers of their product platform are incentivized to productize and list some content of their business practices on their app store. Often those insights are provided without charge. These app stores profit from the community as they can capture value through the ecosystem from a multitude of free offerings. Differing from the other types, the Community Platform also accepts contributions from complementors who are legally acting as individuals rather than companies. Typically, the app store is part of the community portal and access requires a customer or developer account. One of two app stores examined in this category provided a website for the submission of app store products that could be used without requiring an account. The submission page could be accessed without the need to apply or register for any kind of partnership. This is used to decrease the burdens associated with going through onboarding and qualification processes. However, as fewer monetary incentives are involved, the onboarding process is quicker and tied to less stringent criteria than the other types of B2B app stores. Community Platform-based app stores offer APIs with documentation and low-code development environments as part of their core offering. This eases the process of developing and productizing knowledge. In all implementations of this type of app store, input control was kept to a minimum and quality was only assured on a security and technical level, but not on a functional or content level. One app store owner stated that the applications on their platform "are community tested" and if "users have issues they kind of discuss on it." [In1-In10]

\section{Discussion}

As customers of B2B software platforms increasingly rely on third-party solutions that augment the platform's core functionality [47], B2B app stores are rapidly gaining importance. To operate a successful B2B platform, app store owners must be particularly attentive to the specific requirements of complementors and the generally high expectations of customers. Our study aims to provide a detailed understanding of these control concepts in B2B app stores.

There are some limitations to the results of this study. Providing a complete representation all existing B2B app stores was not feasible. However, our study of ten prestigious cases with a multitude of supporting documents supports a substantial body of empirical evidence. Furthermore, some information on control mechanisms is only accessible to commercial customers or users of the platform. We conducted interviews with industry experts to fill this gap. By not assigning companies to the retrieved interview data, we derived three taxonomies that provide a generalist view of the industry. In addition, it may be interesting to conduct a longitudinal study to gain a process view of possible changing B2B app store governance paradigms within this rapidly evolving field. However, we attempted to mitigate this problem by collecting data over seven months.

With our findings on B2B app store governance, we first add to the body of literature on platform ecosystem governance by providing three taxonomies that reveal concrete and tangible dimensions and characteristics on B2B app store governance. Combining findings from research on case study insights and platform governance allows us to enrich the general guidance on governing a digital platform by considering vital characteristics of B2B app stores 
[17]. Differing from other studies that consider an app store as an interface between the platform owner and ecosystem actors, we consider the app store as a "digital storefront" and as an integral part of the underlying platform [2]. This perspective allows us to explore the conceptual integration of both constructs and hence provides grounded insights. For example, we show that existing principles for monetization need to be extended with novel commercial models emerging in B2B app stores, e.g., consumption-based pricing models or upfront commitment of usage.

Second, our results highlight the importance of distinguishing between different manifestations of governance concepts for B2B app stores. Ultimately, we derive three robust app store types, each of which provides a different rationale for the interpretation and expression of governance characteristics. All app store types show that they can be distinguished along the derived dimensions in the taxonomies based on the selected characteristics. An important dimension that helps distinguish app store types is complementary management, as some app stores provide extensive support for managing complementors, while others limit dedicated support and rely on the selfsustainability of complementors.

In practice, our findings provide insights for platform owners that guide the implementation and operation of B2B app stores. Specifically, we derive three relevant lessons learned from the study: First, conveying the platforms' identity to users and complementors is a crucial factor in increasing overall engagement and usage [In7]. Complementors that develop, list, and market their solutions in the same fashion as the platform owner primarily create solutions aligned with the platform's core principles, which ultimately lead to higher user satisfaction. Platform owners address this with a business plan that complementors must follow when registering. Second, offering free and publicly accessible development tools, documentation, and enablement assets is essential to attract complementors [In2]. This openness allows complementors to discover integration options and does not create any financial obligations in the exploration and development phase. Third, while ensuring the overall rigor of the applications, platform owners limit quality assurance to functional and security levels while leaving other levels unverified [In8]. App store owners facilitate this by having customers review the solutions they procure, as they have extensive industry experience and can provide detailed and use case-specific insights into the applications. It also favors user-to-user communication, as users tend to have similar backgrounds and can discuss the quality of solutions on a similar level. This trend can be seen in the emergence of third-party platforms for B2B software evaluation, e.g., G2 Crowd or Gartner Peer Insights.

\section{References}

[1] Salesforce Inc. The History of Salesforce. 2021 [May 29, 2021]; Available from: https://sforce.co/3fJHKci.

[2] R. M. Müller, B. Kijl, and J. K. J. Martens, A Comparison of Inter-Organizational Business Models of Mobile App Stores: There is more than Open vs. Closed. Journal of Theoretical and Applied Electronic Commerce Research, 2011. 6(2): p. 63-76.

[3] T. Al-Naeem, F. Rabhi, B. Benatallah, and P. Ray, Systematic Approaches for Designing B2B Applications. International Journal of Electronic Commerce, 2005. 9(2): p. 41-70.

[4] Celonis SE. EMS Store. 2021 [May 29, 2021]; Available from: https://bit.ly/3uN4QTE.

[5] S. Borg and P. Freytag, Helicopter view: An interpersonal relationship sales process framework. Journal of Business \& Industrial Marketing, 2012. 27(7): p. $564-571$.

[6] G. Dushnitsky, E. Piva, and C. Rossi-Lamastra, Investigating the Mix of Strategic Choices and Performance of Transaction Platforms: Evidence from the Crowdfunding Setting. Strategic Management Journal, 2020: p. 1-36.

[7] AWS Inc. What's New with AWS Marketplace? 2021 [May 15, 2021]; Available from: https://amzn.to/2SQ9Klo.

[8] D. McIntyre and A. Srinivasan, Networks, platforms, and strategy: Emerging views and next steps. Strategic Management Journal, 2016. 38(1): p. 141-160.

[9] F. F. Suarez and M. A. Cusumano, The Role of Services in Platform Markets, in Platforms, Markets and Innovation. 2009, Edward Elgar Publishing: Cheltenham, United Kingdom.

[10] K. Boudreau, Open Platform Strategies and Innovation: Granting Access vs. Devolving Control. Management Science, 2010. 56(10): p. 1849-1872.

[11] A. Ghazawneh and O. Henfridsson, Balancing platform control and external contribution in third-party development: The boundary resources model. Information Systems Journal, 2013. 23(2): p. 173-192.

[12] R. Floetgen, M. Novotny, F. Urmetzer, and M. Böhm, Connecting the Dots of Digital Platform Ecosystem Research: Constructs, Causal Links and Future Research, in ECIS Research Papers. 53. 2021: Marrakesah, Morocco.

[13] A. Ghazawneh and O. Henfridsson, A paradigmatic analysis of digital application marketplaces. Journal of Information Technology, 2015. 30(3): p. 198-208.

[14] A. Hein, M. Schreieck, M. Wiesche, and H. Krcmar, Multiple-Case Analysis on Governance Mechanisms of Multi-Sided Platforms, in Multikonferenz Wirtschaftsinformatik 2016: Ilmenau, Germany.

[15] G. Perscheid, N. Ostern, and J. Moormann. Determining Platform Governance: Framework for Classifying Governance Types. in In proceedings of the 41st 
International Conference on Information Systems (ICIS). 2020. Virtual Conference.

[16] R. Nickerson, U. Varshney, and J. Muntermann, $A$ Method for Taxonomy Development and its Application in Information Systems. European Journal of Information Systems, 2013. 22(3): p. 336-359.

[17] M. Schreieck, M. Wiesche, and H. Krcmar, Design and Governance of Platform Ecosystems - Key Concepts and Issues for Future Research, in 24th European Conference on Information Systems (ECIS). 2016: Istanbul, Turkey.

[18] R. J. Floetgen, J. Strauss, J. Weking, A. Hein, F. Urmetzer, M. Böhm, and H. Krcmar, Introducing platform ecosystem resilience: leveraging mobility platforms and their ecosystems for the new normal during COVID-19. European Journal of Information Systems, 2021. 30(3): p. 304-321.

[19] A. Tiwana, Platform Ecosystems. Aligning Architecture, Governance and Strategy. 2014, Waltham, MA, USA: Elsevier Inc.

[20] A. Hein, M. Schreieck, T. Riasanow, D. S. Setzke, M. Wiesche, M. Böhm, and H. Krcmar, Digital platform ecosystems. Electronic Markets, 2019. 30(1): p. 87-98.

[21] R. Floetgen, S. Gomm, M. Böhm, and H. Krcmar, Implications of Emerging Financial Regulatory Reporting Frameworks for Digital Platforms Boundary Resources, in AMCIS Proceedings. 2. 2020: Salt Lake City, USA.

[22] M. Amberg, I. Thiessen, M. Lang, and B. Belkius, Mobile Application Marketplaces - an Investigation from Customers' Perspective, in Multikonferenz Wirtschaftsinformatik. 2010: Göttingen, Germany.

[23] J. Kim, I. Kim, and H. Lee, The Success Factors for App Store-Like Platform Businesses from the Perspective of Third-Party Developers: An Empirical Study Based on A Dual Model Framework, in Pacific Asia Conference on Information Systems (PACIS). 2010: Taipei, Taiwan. p. $272-283$

[24] T. Hurni and T. Huber, The Interplay of Power and Trust in Platform Ecosystems of the Enterprise Application Software Industry, in 22nd European Conference on Information Systems (ECIS). 2014: Tel Aviv, Israel.

[25] A. Ghose and S. P. Han, Estimating Demand for Mobile Applications in the New Economy. Management Science, 2014. 60(6): p. 1470-1488.

[26] Amazon Web Services Inc. AWS Marketplace - Seller Guide. 2020 [February 13, 2021]; Available from: https://amzn.to/3fHrxEw.

[27] T. F. Bresnahan and S. Greenstein, Technological Competition and the Structure of the Computer Industry. The Journal of Industrial Economics, 1999. 47(1): p. 140.

[28] A. Ghazawneh and O. Mansour, Value Creation in Digital Application Marketplaces: A Developer's Perspective, in The 36th International Conference on Information Systems (ICIS). 2015: Fort Worth, USA.

[29] M. Wiesche, C. Berwing, M. Schermann, and H. Krcmar. Patterns for Understanding Control Requirements for Information Systems for Governance, Risk Management, and Compliance (GRC IS). in
Advanced Information Systems Engineering Workshops. 2011. Berlin, Heidelberg: Springer Berlin Heidelberg.

[30] Salesforce Inc. AppExchange Partner Program Benefits Value Proposition. 2021 [March 16, 2021]; Available from: https://sforce.co/3cb5Iv4.

[31] T. Goldbach and V. Kemper, Should I stay or should I go? The effects of control mechanisms on app developers' intention to stick with a platform, in 22nd European Conference on Information Systems (ECIS). 2014: Tel Aviv, Israel.

[32] B. Bergvall-Kåreborn and D. Howcroft, Mobile Applications Development on Apple and Google Platforms. Communications of the Association for Information Systems, 2011. 29(30): p. 565-580.

[33] T. Goldbach, V. Kemper, and A. Benlian, Mobile application quality and platform stickiness under formal vs. self-control - Evidence from an experimental study. 35th International Conference on Information Systems (ICIS). 2014, Auckland, New Zealand.

[34] J. Corbin and A. Strauss, Basics of qualitative research: Techniques and procedures for developing grounded theory, 3rd ed. 2008, Thousand Oaks, CA, US: Sage Publications, Inc.

[35] G. Punj and D. Steward, Cluster analysis in marketing research: Review and suggestions for application. Journal of Marketing Research, 1983. 20(2): p. 134-148.

[36] R. K. Yin, Case Study Research Design and Methods (5th ed.). 2014, Thousand Oaks, California, USA: Sage Publications.

[37] R. E. Stake, The Art of Case Study Research. 1995, Thousand Oaks, CA, USA: Sage Publications.

[38] J. Gläser and G. Laudel, Experteninterviews und qualitative Inhaltsanalyse. 2010, Heidelberg, Berlin: Springer-Verlag.

[39] Appian Inc. Appian Partner Program Guide. 2020 [May 23, 2021]; Available from: https://bit.ly/3g8SuAa.

[40] Microsoft Inc. Microsoft App Source \& Azure Marketplace - Best Practices Guide. 2020 [March 16, 2021]; Available from: https://bit.ly/34ERuOF.

[41] Blue Prism Ltd. Blue Prism Digital Exchange. 2020 [May 12, 2021]; Available from: https://bit.ly/3vJcYpJ.

[42] Celonis SE. Meet the Execution Management System. 2021 [May 29, 2021]; Available from: https://bit.ly/3wLf6gs.

[43] Pega Inc. Pega program guide for independent software vendors (ISVS). 2018 [March 16, 2021]; Available from: https://pe.ga/3pcYCeu.

[44] SAP SE. Design, innovate, and market solutions with SAP. 2019 [March 20, 2021]; Available from: https://bit.ly/3pbdaLV.

[45] ServiceNow Inc. Technology Partner Program. 2020 [March 13, 2021]; Available from: https://bit.ly/3uDBhnD.

[46] Splunk Inc. Splunk Add-on Builder User Guide 3.0.1. 2021 [March 13, 2021]; Available from: https://splk.it/2SQzRsJ.

[47] R. Floetgen, N. Mitterer, F. Urmetzer, and M. Böhm, Platform Ecosystem Structures: Leveraging Platformbased Technology and the Finance Ecosystem for the New Normal, in PACIS Proceedings. 180. 2021: Dubai, UAE. 